\title{
Diagnostic dilemma regarding the extent of implantable cardioverter-defibrillator infection: Pocket infection alone or pocket infection with concomitant lead-dependent infective endocarditis?
}

\author{
Barbara Małecka ${ }^{1,2}$, Andrzej Ząbek ${ }^{1}$, Maciej Dębski ${ }^{1}$, Krzysztof Boczar ${ }^{1}$, \\ Magdalena Kostkiewicz ${ }^{2,3,4}$, Jacek Lelakowski ${ }^{1,2}$ \\ ${ }^{1}$ Department of Electrocardiology, John Paul II Hospital, Krakow, Poland \\ ${ }^{2}$ Institute of Cardiology, Jagiellonian University Medical College, Krakow, Poland \\ ${ }^{3}$ Nuclear Medicine Department, John Paul II Hospital, Krakow, Poland \\ ${ }^{4}$ Cardiovascular Diseases Department, Jagiellonian University Medical College, Krakow, Poland
}

A 57-year-old man with dual-chamber implantable cardioverter-defibrillator (ICD), 13 years after implantation, was referred for transvenous lead extraction (TLE) due to pocket infection. ICD pocket site showed hyperthermia, redness, swelling, pain and purulent skin fistula. Inflammatory blood markers were normal and serial blood cultures were negative. Transthoracic and transesophageal echocardiography showed a mass on the ventricular lead in the right atrium, which was $16 \times 15 \mathrm{~mm}$ in diameter (Fig. 1).

Presence of vegetation and local infection are major Duke criteria for diagnosis of lead-dependent infective endocarditis (LDIE). Therefore, it was crucial to clarify whether the intracardiac mass represented a vegetation. The radionuclide-labeled

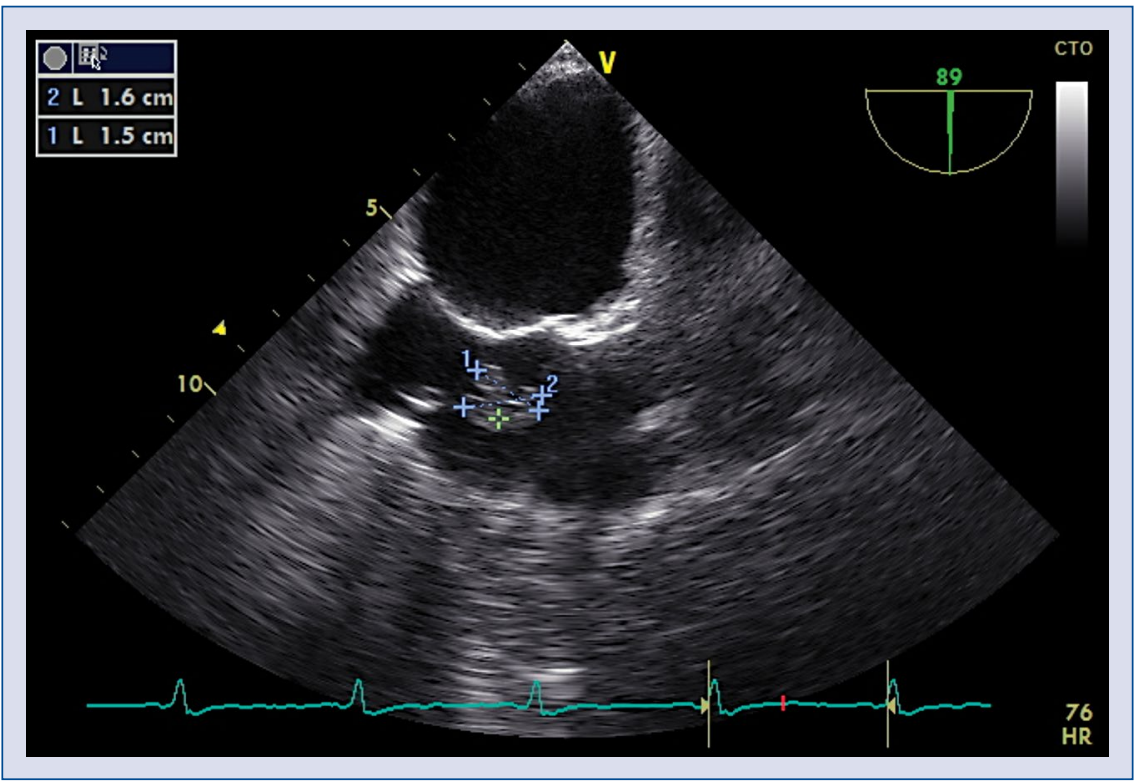

Figure 1. Transesophageal echocardiogram — see text for details.

Address for correspondence: Maciej Dębski, MD, Department of Electrocardiology, John Paul II Hospital, ul. Prądnicka 80, 31-202 Kraków, Poland, tel: +48 12 6142277, fax: +48 12 6332399, e-mail: maciekdebski@gmail.com

Received: 10.06.2017 Accepted: 01.11.2017 
white blood cell scintigraphy (WBC SPECT-CT) showed increased tracer uptake only in ICD pocket. After the successful TLE procedure the patient received a 2 -week course of ciprofloxacin orally directed against methicillin-sensitive staphylococcus aureus culture obtained from ICD pocket, and enoxaparin treatment. After the wound was healed the patient was admitted to hospital again and dual-chamber ICD was implanted on the right side of the chest. Patient attended an outpatient clinic and at 13 months had no signs of infection relapse.

This case highlights the difficulties in obtaining a definitive and correct diagnosis in patients with cardiac implantable electronic device related infection. A lead-associated mass does not necessarily mean that it is vegetation of lead-dependent infective endocarditis. Importantly, thrombus formation is a frequent finding several years after implantation. A rash decision to diagnose LDIE would have resulted in a much longer course of antibiotic treatment, hospitalization and postponing of ICD reimplantation. WBC SPECT-CT imaging played a major role in diagnostic work-up as it helped in a differential diagnosis in a patient with undefined intracardiac mass and led to a short term antibiotic treatment, together with the use of anticoagulation treatment.

Conflict of interest: None declared 